\title{
Invariant maximal positive subspaces and polar decompositions
}

\author{
Christian Mehl * André C. M. Ran ${ }^{\dagger} \quad$ Leiba Rodman ${ }^{\ddagger}$
}

\begin{abstract}
It is proved that invertible operators on a Krein space which have an invariant maximal uniformly positive subspace and map its orthogonal complement into a nonnegative subspace allow polar decompositions with additional spectral properties. As a corollary, several classes of Krein space operators are shown to allow polar decompositions. An example in a finite dimensional Krein space shows that there exist dissipative operators that do not allow polar decompositions.
\end{abstract}

AMS Subject classification: Primary 47B44, 46C20

Keywords: Krein space, invariant subspace, dissipative operator, polar decomposition

\section{Introduction and main result}

Let $\mathcal{H}$ be a (complex) Hilbert space with the inner product $\langle\cdot, \cdot\rangle$, and let $J$ be an invertible (bounded) selfadjoint operator on $\mathcal{H}$. The operator $J$ induces a Krein space structure on $\mathcal{H}$ in a standard way: The generally indefinite inner product on $\mathcal{H}$ is defined by $[x, y]=$ $\langle J x, y\rangle, x, y \in \mathcal{H}$. A closed (in the topology induced by $\langle\cdot, \cdot\rangle$ ) subspace $\mathcal{M}$ of $\mathcal{H}$ is called uniformly $J$-positive if $[x, x] \geq \epsilon\langle x, x\rangle$ for every $x \in \mathcal{M}$, where $\epsilon>0$ is independent of $x$. A uniformly $J$-positive subspace is called maximal uniformly $J$-positive if no strictly larger subspace of $\mathcal{H}$ is uniformly $J$-positive. For example, the spectral subspace of $J$ corresponding to the positive part of the spectrum of $J$ is maximal uniformly $J$-positive. The reader is referred to the books [1], [3], [2], [10] (finite dimensional Krein spaces only), [11] for information on geometry and classes of linear operators in Krein spaces.

*Fakultät II; Institut für Mathematik, Technische Universität Berlin, D-10623 Berlin, Germany, Email: mehl@math.tu-berlin.de

†Afdeling Wiskunde, Faculteit der Exacte Wetenschappen, Vrije Universiteit Amsterdam, De Boelelaan 1081a, 1081 HV Amsterdam, The Netherlands, Email: ran@cs.vu.nl

${ }^{\ddagger}$ College of William and Mary, Department of Mathematics, P.O.Box 8795, Williamsburg, VA 231878795, USA, Email: Ixrodm@math.wm. edu. The research of the third author was partially supported by the NWO during his visit at the Vrije Universiteit, and by the Faculty Research Assignment of the College of William and Mary. 
All operators on $\mathcal{H}$ are assumed to be linear and bounded (with respect to the Hilbert norm $\|x\|=\sqrt{\langle x, x\rangle}$ ). The adjoint operator $Y^{[*]}$ of an operator $Y$ with respect to $J$ is defined by $[Y x, y]=\left[x, Y^{[*]} y\right], x, y \in \mathcal{H}$; the Hilbert space adjoint will be denoted $Y^{*}$. An operator $Y$ on $\mathcal{H}$ is called $J$-selfadjoint if $Y=Y^{[*]}$, and $J$-unitary if $Y$ is invertible and $Y^{-1}=Y^{[*]}$. If $\mathcal{M} \subseteq \mathcal{H}$ is a subspace (all subspaces are assumed to be closed), then we denote by $\mathcal{M}^{[\perp]}$ the orthogonal companion of $\mathcal{M}$, i.e., the subspace formed by the vectors $J$-orthogonal to $\mathcal{M}$.

A $J$-polar decomposition of an operator $X$ is a decomposition of the form $X=U A$, where $U$ is $J$-unitary and $A$ is $J$-selfadjoint. A particular kind of $J$-polar decompositions, involving the notion of $J$-modulus, was introduced in [14], [15]. Recently, polar decompositions in finite dimensional Krein spaces were studied in [7], [4], [5], [6], [12], and in $\Pi_{\kappa}$ spaces in [13]. In contrast with the Hilbert space case, there exist operators already on a 2-dimensional Krein space that do not admit a $J$-polar decomposition.

Of particular interest are $J$-polar decompositions in which the operator $A$ has additional spectral properties. For example, the spectrum of $J$-modulus is assumed to be positive. In the finite dimensional case, if a $J$-polar decomposition exists, one can always choose $A$ to have its spectrum in the closed right halfplane (this follows easily from the results in [5]).

In this paper we prove the following result. It asserts existence and uniqueness of a $J$-polar decomposition of $X$ with the spectrum of $A$ located in a quarterplane centered about the positive half-axis, provided $X$ has an invariant subspace that satisfies certain geometric conditions.

Theorem 1.1 Let $X$ be an invertible operator on $\mathcal{H}$, and suppose that $X$ has an invariant maximal uniformly $J$-positive subspace $\mathcal{M}$ such that $X\left(\mathcal{M}^{[\perp]}\right)$ is J-nonpositive. Then $X$ allows a J-polar decomposition $X=U A$ such that

$$
\sigma(A) \subseteq\{z \in \mathbb{C}: \operatorname{Re}(z) \geq|\operatorname{Im}(z)|\} \backslash\{0\} .
$$

Moreover, the $J$-polar decomposition $X=U A$ with the property (1.1) is unique.

If in addition, the restriction of $X$ to $\mathcal{M}$ is invertible, and the subspace $X\left(\mathcal{M}^{[\perp]}\right)$ is uniformly $J$-negative, then for the unique J-polar decomposition with (1.1) we actually have

$$
\sigma(A) \subseteq\{z \in \mathbb{C}: \operatorname{Re}(z)>|\operatorname{Im}(z)|\} .
$$

Note that invertibility of $\left.X\right|_{\mathcal{M}}$ follows automatically from that of $X$ if at least one of the two spectral subspaces of $J$ corresponding to the positive part and to the negative part of $\sigma(J)$ is finite dimensional.

The proof is based on a lemma which is independently interesting.

Lemma 1.2 If an invertible operator $X$ is such that $X^{[*]} X$ has no spectrum in the open, resp. closed, left halfplane, then $X$ allows a $J$-polar decomposition $X=U A$ such that (1.1), resp., (1.2), holds true. Moreover, the J-polar decomposition $X=U A$ with the property (1.1), resp., (1.2), is unique. 
Proof Using the functional calculus, define

$$
A=\frac{1}{2 \pi i} \int_{\Gamma} z^{1 / 2}\left(z I-X^{[*]} X\right)^{-1} d z
$$

where $\Gamma$ is a closed simple rectifiable contour that does not intersect the negative semiaxis, contains the spectrum of $X^{[*]} X$ in its interior, and is symmetric with respect to the real axis $(z \in \Gamma$ implies $\bar{z} \in \Gamma)$, and where $z^{1 / 2}$ is the analytic branch of the square root function defined on $\Gamma$ and its interior and such that $z^{1 / 2}>0$ if $z>0$. Then $A^{2}=X^{[*]} X$, and one easily checks that $A$ is $J$-selfadjoint. Moreover, by the spectral mapping theorem (1.1) or (1.2), as the case may be, holds true. Next, we show that $U:=X A^{-1}$ is $J$-unitary. Clearly, $U$ is invertible, and $U U^{[*]}=X A^{-2} X^{[*]}=X\left(X^{[*]} X\right)^{-1} X^{[*]}=I$.

It remains to prove the uniqueness. Let $X=U A$ be a polar decomposition, where $A$ satisfies (1.1). (In particular, this case contains polar decompositions, where $A$ satisfies (1.2).) Then $A^{2}=X^{[*]} X$. Again, let $\Gamma$ be a closed simple rectifiable contour that does not intersect the negative semiaxis, contains the spectrum of $X^{[*]} X$ in its interior, and is symmetric with respect to the real axis and let $z^{1 / 2}$ be the analytic branch of the square root function defined on $\Gamma$ and its interior and such that $z^{1 / 2}>0$ if $z>0$. Define

$$
A_{1}=\frac{1}{2 \pi i} \int_{\Gamma} z^{1 / 2}\left(z I-X^{[*]} X\right)^{-1} d z=\frac{1}{2 \pi i} \int_{\Gamma} z^{1 / 2}\left(z I-A^{2}\right)^{-1} d z .
$$

Now

$$
\left(z-A^{2}\right)^{-1}=\frac{1}{2} A^{-1}\left(\left(z^{1 / 2}-A\right)^{-1}-\left(z^{1 / 2}+A\right)^{-1}\right) .
$$

So,

$$
A A_{1}=\frac{1}{4 \pi i}\left(\int_{\Gamma} z^{1 / 2}\left(z^{1 / 2}-A\right)^{-1} d z-\int_{\Gamma} z^{1 / 2}\left(z^{1 / 2}+A\right)^{-1} d z\right) .
$$

We substitute $z^{\frac{1}{2}}=t$, and define $\Gamma^{\prime}=\left\{z^{\frac{1}{2}} \mid z \in \Gamma\right\}$. Then $z=t^{2}$ on $\Gamma$ with $t \in \Gamma^{\prime}$, and substitution gives

$$
A A_{1}=\frac{1}{2 \pi i}\left(\int_{\Gamma^{\prime}} t^{2}(t-A)^{-1} d t-\int_{\Gamma^{\prime}} t^{2}(t+A)^{-1} d t\right) .
$$

Since the real part of $t$ is nonnegative on $\Gamma^{\prime}$, we have that $\sigma(-A)$ is in the exterior of $\Gamma^{\prime}$. So the second integral above is zero, as the integrand is analytic inside $\Gamma^{\prime}$. Hence

$$
A A_{1}=\frac{1}{2 \pi i} \int_{\Gamma^{\prime}} t^{2}(t-A)^{-1} d t
$$

Now since $\sigma\left(A^{2}\right)$ is contained in the interior of $\Gamma$ and since $A$ satisfies (1.1), we have that $\sigma(A)$ is contained in the interior of $\Gamma^{\prime}$. Therefore, by the functional calculus of $A$, we have that

$$
A A_{1}=A^{2},
$$

and as $A$ is invertible, it follows that $A=A_{1}$. Thus $A$ is unique, and hence also $U=X A^{-1}$. 
We mention in passing that the uniqueness of $A$ follows also from the following general result concerning a monic operator polynomial $L(\lambda)$ and its monic operator polynomial right divisor $L_{1}(\lambda)$ of degree $k$ (we apply the result with $L(\lambda)=z^{2} I-X^{[*]} X$ and $L_{1}(\lambda)=$ $z I-A)$ : If $\gamma$ is a closed rectifiable contour such that the spectrum of $L_{1}(\lambda)$ is inside $\gamma$ and the spectrum of the operator polynomial $L(\lambda)\left(L_{1}(\lambda)\right)^{-1}$ is outside $\gamma$, then there exists only one operator polynomial right divisor of $L(\lambda)$ with spectrum inside $\gamma$ and the same degree $k$, namely $L_{1}(\lambda)$. This follows easily from the spectral theory of operator polynomials [9], also [17]. For further details we refer the reader to these sources.

Proof (of the theorem). By the lemma we need to show that

$$
\sigma\left(X^{[*]} X\right) \cap\{z \in \mathbb{C}: \operatorname{Re}(z)<0\}=\emptyset .
$$

Write $X$ and $J$ as $2 \times 2$ block operator matrices with respect to the orthogonal decomposition $\mathcal{H}=\mathcal{M} \oplus(\mathcal{M})^{\perp}$ :

$$
X=\left(\begin{array}{cc}
X_{11} & X_{12} \\
0 & X_{22}
\end{array}\right), \quad J=\left(\begin{array}{cc}
J_{11} & J_{12} \\
J_{12}^{*} & J_{22}
\end{array}\right) .
$$

Here, $J_{11}$ is positive definite and invertible. Applying a transformation

$$
X \mapsto P^{-1} X P, \quad J \mapsto P^{*} J P, \quad \text { where } P=\left(\begin{array}{cc}
J_{11}^{-1 / 2} & -J_{11}^{-1} J_{12} \\
0 & I
\end{array}\right),
$$

we can (and will) assume without loss of generality that $J_{11}=I$ and $J_{12}=0$. Since $\mathcal{M}$ is maximal uniformly $J$-positive, the $(2,2)$-block $J_{22}$ is necessarily congruent to $-I$. Thus, we may assume that $X$ and $J$ have the forms

$$
X=\left(\begin{array}{cc}
X_{11} & X_{12} \\
0 & X_{22}
\end{array}\right), \quad J=\left(\begin{array}{cc}
I & 0 \\
0 & -I
\end{array}\right) .
$$

Then one easily computes that

$$
X^{[*]} X=\left(\begin{array}{cc}
X_{11}^{*} X_{11} & X_{11}^{*} X_{12} \\
-X_{12}^{*} X_{11} & X_{22}^{*} X_{22}-X_{12}^{*} X_{12}
\end{array}\right)
$$

As $X$ is invertible, so is $X^{[*]} X$.

Arguing by contradiction, suppose that $X^{[*]} X$ has spectrum in the open left half plane, and let $\lambda \in \mathbb{C}, \operatorname{Re}(\lambda)<0$ be a boundary point of $\sigma\left(X^{[*]} X\right)$. Then $\lambda$ belongs to the approximate point spectrum (see, e.g., [8]), i.e., there is a sequence $\left\{z_{n}=\left(x_{n}, y_{n}\right)\right\}_{n=1}^{\infty}$, $x_{n} \in \mathcal{M}, y_{n} \in(\mathcal{M})^{\perp}$ such that $\left\|z_{n}\right\|=1$ and $\left(X^{[*]} X-\lambda I\right) z_{n} \longrightarrow 0$ as $n \longrightarrow \infty$ :

$$
\begin{aligned}
X_{11}^{*} X_{11} x_{n}+X_{11}^{*} X_{12} y_{n}-\lambda x_{n} & \longrightarrow 0 \\
-X_{12}^{*} X_{11} x_{n}+\left(X_{22}^{*} X_{22}-X_{12}^{*} X_{12}\right) y_{n}-\lambda y_{n} & \longrightarrow 0 .
\end{aligned}
$$

From the fact that $\operatorname{Re}(\lambda)$ is negative, we obtain that $\lambda I-X_{11}^{*} X_{11}$ is invertible and the inverse $\left(\lambda I-X_{11}^{*} X_{11}\right)^{-1}$ has a negative definite and invertible selfadjoint part. Recall that for any operator $X$ on $\mathcal{H}$, the operator $\frac{1}{2}\left(X+X^{*}\right)$ is called the selfadjoint part of $X$. 
We get from (1.6):

$$
x_{n}-\left(\lambda I-X_{11}^{*} X_{11}\right)^{-1} X_{11}^{*} X_{12} y_{n} \longrightarrow 0 \text {. }
$$

Inserting this in (1.7) we obtain

$$
\left(\lambda I-\left(X_{22}^{*} X_{22}-X_{12}^{*} X_{12}\right)+X_{12}^{*} X_{11}\left(\lambda I-X_{11}^{*} X_{11}\right)^{-1} X_{11}^{*} X_{12}\right) y_{n} \longrightarrow 0 .
$$

We set

$$
F(\lambda)=\lambda I-\left(X_{22}^{*} X_{22}-X_{12}^{*} X_{12}\right)+X_{12}^{*} X_{11}\left(\lambda I-X_{11}^{*} X_{11}\right)^{-1} X_{11}^{*} X_{12}
$$

The condition that $X\left(\mathcal{M}^{[\perp]}\right)$ is $J$-nonpositive translates into $X_{22}^{*} X_{22}-X_{12} X_{12}^{*}$ being positive semidefinite. It then follows from (1.10) that $F(\lambda)$ has a negative definite and invertible selfadjoint part. In particular, $F(\lambda)$ is invertible.

Hence from (1.9) we see that $y_{n} \longrightarrow 0$. Then (1.8) implies that also $x_{n} \longrightarrow 0$, a contradiction with $\left\|z_{n}\right\|=1$.

The proof of the additional part of Theorem 1.1 follows the same lines. We now have $\operatorname{Re}(\lambda) \leq 0$. The invertibility of $\left.X\right|_{\mathcal{M}}$ implies that $X_{11}^{*} X_{11}$ is invertible, hence again $\lambda I-X_{11}^{*} X_{11}$ is invertible and the inverse $\left(\lambda I-X_{11}^{*} X_{11}\right)^{-1}$ has a negative definite and invertible selfadjoint part. The condition that $X\left(\mathcal{M}^{[\perp]}\right)$ is uniformly $J$-negative means that $X_{22}^{*} X_{22}-X_{12} X_{12}^{*}$ is positive definite invertible. So we conclude again from (1.10) that $F(\lambda)$ is invertible, and obtain a contradiction.

Remark 1.3 The theorem obviously remains true if $\mathcal{M}$ is assumed to be an invariant maximal uniformly $J$-negative subspace of $X$ such that $X\left(\mathcal{M}^{[\perp]}\right.$ is $J$-nonnegative. (Replace $J$ with $-J$ in the theorem.)

\section{Polar decompositions for various classes of opera- tors and examples}

Several consequences of Theorem 1.1 and illustrative examples are presented in this section.

Corollary 2.1 Let $X$ be an invertible operator such that the spectrum of $X$ does not intersect the unit circle, and assume that one of the following two conditions holds:

(a.) the spectrum of $X$ does not intersect the unit circle, and $X$ is strictly monotone; that is, either $[X x, X x]>[x, x]$ for every nonzero $x \in \mathcal{H}$, or $[X x, X x]<[x, x]$ for every nonzero $x \in \mathcal{H}$.

(b.) the spectral subspace of $J$ corresponding to the positive part of $\sigma(J)$ is finite dimensional, and $[X x, X x]>[x, x]$ for every nonzero $x \in \mathcal{H}$ with $[x, x] \geq 0$.

Then $X$ admits a J-polar decomposition with the property (1.1). 
Proof First consider case (a.) Assume that $[X x, X x]>[x, x]$ for every nonzero $x \in \mathcal{H}$, and that the spectrum of $X$ does not intersect the unit circle. The proof of the case $[X x, X x]<[x, x]$ is similar.

According to $\left[11\right.$, Theorem 11.1] there exist two subspaces $\mathcal{H}_{-}$and $\mathcal{H}_{+}$which are $X$ invariant and maximal $J$-negative, respectively, maximal $J$-positive, and for which we have the direct sum decomposition $\mathcal{H}=\mathcal{H}_{+} \dot{+} \mathcal{H}_{-}$. Observe that this direct sum decomposition is not necessarily $J$-orthogonal. Note that the statements cited from [11] are made for the case of $\Pi_{\kappa}$ spaces, that is, for spaces for which the spectral subspace of $J$ corresponding to the positive part of $\sigma(J)$ is finite dimensional. However, the proof given there carries over directly to the general case, as is already remarked in [11] (Note 2 on page 80).

According to $\left[1\right.$, Theorem 5.2] the spaces $\mathcal{H}_{-}$and $\mathcal{H}_{+}$are uniformly $J_{\text {-negative, respec- }}$ tively, uniformly $J$-positive. In order to be able to apply Theorem 1.1, we will establish that $X\left(\mathcal{H}_{-}^{[\perp]}\right)$ is $J$-nonnegative. Then in view of Remark 1.3, we can apply Theorem 1.1 with "positive" replaced by "negative" everywhere in the statement. So, let $x \in \mathcal{H}_{-}^{[\perp]} \backslash\{0\}$. According to [3, Lemma I.6.3] the space $\mathcal{H}_{-}^{[\perp]}$ is $J$-nonnegative. So, $[x, x] \geq 0$. Since $[X x, X x]>[x, x]$ it follows that $X x$ is a $J$-positive vector. Hence $X\left(\mathcal{H}_{-}^{[\perp]}\right)$ is $J$-nonnegative.

In case (b.), the result follows in the same way, but using [11, Theorem 11.4] instead of [11, Theorem 11.1].

It is known that in finite dimensional Krein spaces strictly monotone operators always allow J-polar decompositions, see [14], [4, Theorem 2.4].

Example 2.2 Let $\lambda>0, \varepsilon= \pm 1$ and consider

$$
J=\varepsilon\left(\begin{array}{cc}
0 & 1 \\
1 & 0
\end{array}\right), \quad X=\varepsilon\left(\begin{array}{cc}
-i \lambda & \frac{i}{2 \lambda} \\
0 & i \lambda
\end{array}\right) .
$$

Then

$$
i\left(X^{*} J-J X\right)=\left(\begin{array}{cc}
0 & 0 \\
0 & \frac{1}{\lambda}
\end{array}\right),
$$

so that $X$ is $J$-dissipative. Recall that $X$ is $J$-dissipative if $\frac{1}{2 i}\left(J X-X^{*} J\right)$ is a positive semidefinite matrix. If $X$ were to admit a $J$-polar decomposition, then $X^{[*]} X$ would be the square of the $J$-selfadjoint factor. However,

$$
X^{[*]} X=\left(\begin{array}{cc}
-\lambda^{2} & 1 \\
0 & -\lambda^{2}
\end{array}\right)
$$

and this does not have a $J$-selfadjoint square root (see also [5, Theorem 4.4]). We conclude that not every $J$-dissipative operator admits a $J$-polar decomposition.

Recall that a $J$-dissipative operator in a finite dimensional Krein space always has an invariant maximal $J$-nonnegative subspace (see, e.g., [16]). In Example 2.2, the $X$-invariant maximal $J$-nonnegative subspaces are

$$
\mathcal{M}_{1}:=\operatorname{Span}\left(\begin{array}{l}
1 \\
0
\end{array}\right), \quad \varepsilon= \pm 1
$$


and

$$
\mathcal{M}_{2}:=\operatorname{Span}\left(\begin{array}{c}
1 \\
4 \lambda^{2}
\end{array}\right), \quad \varepsilon=1
$$

Clearly, $\mathcal{M}_{1}^{[\perp]}=\mathcal{M}_{1}$, hence $X\left(\mathcal{M}_{1}^{[\perp]}\right)=\mathcal{M}_{1}$ is $J$-nonpositive.

Thus, we cannot replace the condition that $X$ has an invariant maximal uniformly $J$-positive subspace in Theorem 1.1 by the condition that $X$ has an invariant maximal $J$-nonnegative subspace, not even in the finite dimensional case.

Example 2.3 Let

$$
J=\left(\begin{array}{cc}
1 & 0 \\
0 & -1
\end{array}\right), \quad B=i\left(\begin{array}{cc}
1 & \alpha \\
0 & -1
\end{array}\right), \quad \alpha \in \mathbb{C}, \quad|\alpha| \leq 2 .
$$

Then $B$ is strictly $J$-dissipative, i.e., $i\left(B^{*} J-J B\right)$ is positive definite, for $|\alpha|<2$ and $J$-dissipative, i.e., $i\left(B^{*} J-J B\right)$ is positive semidefinite, for $|\alpha| \leq 2$. Moreover,

$$
B^{[*]} B=\left(\begin{array}{cc}
1 & \alpha \\
-\bar{\alpha} & 1-|\alpha|^{2}
\end{array}\right) .
$$

One easily checks that this matrix has the eigenvalues

$$
1-\frac{1}{2}|\alpha|^{2} \pm \frac{1}{2} \sqrt{|\alpha|^{4}-4|\alpha|^{2}}
$$

Thus, $B^{[*]} B$ has no eigenvalues on the negative half axis for $|\alpha|<2$ and hence, $B$ does admit $J$-polar decomposition by the results in [5].

Take $\mathcal{M}_{1}=\operatorname{Span}\left(\begin{array}{l}1 \\ 0\end{array}\right)$. Then $\mathcal{M}_{1}$ is a $B$-invariant maximal uniformly $J$-positive subspace. Then

$$
B\left(\mathcal{M}_{1}^{[\perp]}\right)=B\left(\operatorname{Span}\left(\begin{array}{c}
0 \\
1
\end{array}\right)\right)=\operatorname{Span}\left(\begin{array}{c}
\alpha \\
-1
\end{array}\right) .
$$

Clearly this is $J$-nonpositive only if $|\alpha| \leq 1$. So, for the case $|\alpha| \leq 1$ Theorem 1.1 applies and asserts unique existence of a $J$-polar decomposition $B=U A$, where $A$ satisfies (1.1) or (1.2).

However, for $1<|\alpha|<2$ Theorem 1.1 does not apply, not even in the version with "positive" replaced by "negative" everywhere in the statement. Indeed, consider $\mathcal{M}_{2}=$ $\operatorname{Span}\left(\begin{array}{c}\alpha \\ -2\end{array}\right)$. Then $\mathcal{M}_{2}$ is a $B$-invariant maximal uniformly $J$-negative subspace, and

$$
B\left(\mathcal{M}_{2}^{[\perp]}\right)=B\left(\operatorname{Span}\left(\begin{array}{c}
2 \\
-\bar{\alpha}
\end{array}\right)\right)=\operatorname{Span}\left(\begin{array}{c}
|\alpha|^{2}-2 \\
-\bar{\alpha}
\end{array}\right)
$$

This space is $J$-negative for $1<|\alpha|<2$, because

$$
\left(|\alpha|^{2}-2,-\alpha\right)\left(\begin{array}{cc}
1 & 0 \\
0 & -1
\end{array}\right)\left(\begin{array}{c}
|\alpha|^{2}-2 \\
-\bar{\alpha}
\end{array}\right)=|\alpha|^{4}-5|\alpha|^{2}+4
$$


which is negative for the indicated values of $\alpha$. Observe that for $\sqrt{2} \leq|\alpha|<2$ the eigenvalues of $B^{[*]} B$ are located in the open left half plane, so $B$ cannot have a $J$-polar decomposition $B=U A$ such that $A$ satisfies (1.1) or (1.2). However, $B$ still admits a $J$ polar decomposition. When $|\alpha|=2, B$ is only $J$-dissipative, but not strictly $J$-dissipative. In this case $B^{[*]} B$ is similar to a Jordan block of size 2 associated with the eigenvalue -1 . Hence $B$ does not allow a $J$-polar decomposition because $B^{[*]} B$ does not have a $J$-selfadjoint square root. Again see also [5, Theorem 4.4].

The last observation in Example 2.3 gives rise to the following open question.

Problem 1 Does any strictly J-dissipative operator allow a J-polar decomposition?

The following result can be seen quite quickly as a corollary from our main theorem (although a more direct approach is possible as well, which in the finite dimensional case is probably more straightforward).

Corollary 2.4 Assume that $X$ is invertible and commutes with a uniformly positive operator, that is $X Y=Y X$ for some $J$-selfadjoint $Y$ satisfying $J Y \geq \varepsilon I>0$, where $\varepsilon>0$. Then $X$ admits a J-polar decomposition with the property (1.1).

Proof From [3, Theorem VIII.1.2] it follows that $X$ is fundamentally reducible. Let $\mathcal{M}_{+}$ and $\mathcal{M}_{-}$be a fundamentally reducing pair of subspaces, i.e., they are both $X$-invariant, they are uniformly $J$-positive and uniformly $J$-negative respectively, and $\mathcal{H}=\mathcal{M}_{+}[\dot{+}] \mathcal{M}_{-}$, where this is a $J$-orthogonal direct sum decomposition. Hence, we can apply Theorem 1.1 to get the desired result.

\section{References}

[1] T. Ando. Linear Operators on Krĕ̌n Spaces. Hokkaido University, Research Institute of Applied Electricity, Division of Applied Mathematics, Sapporo, Japan, 1979.

[2] T. Ya. Azizov and I. S. Iohvidov. Linear Operators in Spaces with an Indefinite Metric. John Wiley and Sons, Ltd., Chichester, 1989. (Translated from Russian.)

[3] J. Bognár. Indefinite Inner Product Spaces. Springer-Verlag, New York-Heidelberg, 1974.

[4] Y. Bolshakov, C. V. M. van der Mee, A. C. M. Ran, B. Reichstein, and L. Rodman. Polar decompositions in finite dimensional indefinite scalar product spaces: special cases and applications. Recent developments in operator theory and its applications. Oper. Theory Adv. Appl. (I. Gohberg, P. Lancaster, P. N. Shivakumar, eds.) 87:61-94, 1996, Birkhäuser, Basel. Errata, Integral Equations and Operator Theory, 17:497-501, 1997. 
[5] Y. Bolshakov, C. V. M. van der Mee, A. C. M. Ran, B. Reichstein, and L. Rodman. Polar decompositions in finite dimensional indefinite scalar product spaces: general theory. Linear Algebra Appl. 261:91-141, 1997.

[6] Y. Bolshakov, C. V. M. van der Mee, A. C. M. Ran, B. Reichstein, and L. Rodman. Extensions of isometries in finite dimensional indefinite scalar product spaces and polar decompositions. SIAM J. of Matrix Analysis and Applications, 18:752-774, 1997.

[7] Y. Bolshakov and B. Reichstein. Unitary equivalence in an indefinite scalar product: an analogue of singular-value decomposition. Linear Algebra Appl. 222:155-226, 1995.

[8] J. B. Conway. A Course in Functional Analysis, 2-nd edition. Springer-Verlag, New York, 1990.

[9] I. Gohberg, P. Lancaster, and L. Rodman. Representations and divisibility of operator polynomials. Canad. J. Math., 30: 1045-1069, 1978.

[10] I. Gohberg, P. Lancaster, and L. Rodman. Matrices and Indefinite Scalar Products. Birkhäuser Verlag, Basel, Boston, Stuttgart, 1983.

[11] I. S. Iohvidov, M. G. Kreŭn, and H. Langer. Introduction to the Spectral Theory of Operators in Spaces with an Indefinite Metric. Mathematical Research 9, AkademieVerlag, Berlin, 1982.

[12] C. V. M. van der Mee, A. C. M. Ran, and L. Rodman. Stability of self-adjoint square roots and polar decompositions in indefinite scalar product spaces. Linear Algebra Appl., 302-303:77-104, 1999.

[13] C. V. M. van der Mee, A. C. M. Ran, and L. Rodman. Polar decompositions and related classes of operators in spaces $\Pi_{\kappa}$. Integral Equations and Operator Theory, 44: 50-70, 2002.

[14] V. P. Potapov. A theorem on the modulus, I. Main concepts. The modulus. Theory of Functions, Functional Analysis and its Applications, 38:91-101, 1982, Kharkov (in Russian); English transl.: AMS Translations, Series 2, 138:55-65, 1988.

[15] V. P. Potapov. A theorem on the modulus. II. Theory of Functions, Functional Analysis and its Applications, 39:95-106, 1983, Kharkov (in Russian); English transl.: $A M S$ Translations, Series 2, 138:67-77, 1988.

[16] A. C. M. Ran and D. Temme. Dissipative matrices and invariant maximal semidefinite subspaces, Linear Algebra and its Applications 212/213:169-214, 1994.

[17] L. Rodman. An Introduction to Operator Polynomials, OT38, Birkhäuser Verlag, Basel, 1989. 